\title{
How magnetotactic bacteria make magnetosomes queue up
}

\author{
Richard B. Frankel ${ }^{1}$ and Dennis A. Bazylinski ${ }^{2}$ \\ ${ }^{1}$ Department of Physics, California Polytechnic State University, San Luis Obispo, CA 93407, USA \\ ${ }^{2}$ Department of Biochemistry, Biophysics and Molecular Biology, lowa State University, Ames, IA 50011, USA
}

\begin{abstract}
Magnetotactic bacteria contain chains of magnetosomes that comprise a permanent magnetic dipole in each cell. In two separate, recent papers, Scheffel et al. and Komeili et al. describe the roles of the proteins MamJ and MamK in magnetosome chain formation. Here, we describe the two studies and highlight questions that must be addressed in future investigations of how magnetotactic bacteria construct their magnetic compass needles.
\end{abstract}

\section{Bacterial compasses - the magnetotactic bacteria}

Magnetotactic bacteria are a diverse group of aquatic prokaryotes that align and swim along geomagnetic field lines, a phenomenon called magnetotaxis [1] (Figure 1a). All magnetotactic bacteria contain magnetosomes, which are intracellular iron-mineral crystals within membrane vesicles [2] (Figure 1b and Figure 1c). In most strains, the mineral is magnetite, $\mathrm{Fe}_{3} \mathrm{O}_{4}$, although some marine species biomineralize greigite $\left(\mathrm{Fe}_{3} \mathrm{~S}_{4}\right)$. The crystal sizes, compositions and shapes are remarkably consistent within each bacterial species or strain [2]. Mature crystals are constrained to $\sim 35-120 \mathrm{~nm}$ in diameter, which is within the permanent, single-magnetic-domain size range. This means that each crystal is a tiny permanent magnet. In most species, magnetosomes are arranged in a chain or chains, resulting in a cell magnetic dipole that is the sum of the individual magnetosome dipoles. Because the chain is fixed in position within the cell, the cell passively orients in the magnetic field as it swims, which causes the cell to behave as a self-propelled magnetic compass needle that migrates along geomagnetic field lines. Magnetotaxis is useful to bacteria because it seems to increase their efficiency in finding and maintaining position at a preferred concentration of oxygen in vertical oxygen concentration gradients in aquatic environments [3].

\section{How do bacteria make magnetosomes?}

A key question regarding magnetotactic bacteria since their discovery is how they form and organize their magnetosomes. Because magnetosome crystals have features that are consistent with a biologically-controlled mineralization process [2], it was predicted early on that magnetosome synthesis was under genetic control [1]. When bacteria were subsequently found to produce both $\mathrm{Fe}_{3} \mathrm{O}_{4}$ and $\mathrm{Fe}_{3} \mathrm{~S}_{4}$ crystals, each with a different morphology within the same chain, it was suggested that magnetosome chain formation is also under genetic control and involves different genes than in magnetosome synthesis [4]. However, the genes involved in biomineralization and magnetosome chain formation and regulation were not known. Research progress was hampered by the fastidiousness and microaerophilic nature of magnetotactic bacteria, which makes them difficult to isolate and grow in pure culture. However, there have been several recent important developments in this area of research. First, tractable genetic systems have been devised forMagnetospirillum gryphiswaldense [5] and Magnetospirillum magneticum strain AMB-1 [6]. Second, the genomes of three magnetotactic bacteria, Magnetospirillum magnetotacticum (Joint Genome Institute, http://genome. jgi-psf.org/draft_microbes/magma/magma.home.html), M. magneticum strain AMB-1 [7] and strain MC-1 (Joint Genome Institute, http://genome.jgi-psf.org/draft microbes/magm $1 /$ magm1.home.html), were at least partially sequenced and annotated. Both developments have led to the identification of a large genomic island that contains many of the genes suspected to be involved in magnetosome formation and positioning in the cell $[8,9]$. These genes, the mam genes, encode proteins that are associated with the magnetosome membrane and are organized in clusters within the island $[10,11]$. The mam $A B$ gene cluster is thought to be essential for magnetite production and localization of the magnetosomes. Two genes within this cluster, mamJ and mamK, encode an acidic protein and an actin-like protein that forms filaments in other bacteria, respectively. Finally, new cryogenic techniques have been developed for use in electron tomography that enable visualization of the $3 \mathrm{D}$ internal structure of a bacterium like never before [12].

Construction of the bacterial magnetosome seems to be a complex process that involves several discrete steps including: (i) magnetosome vesicle formation; (ii) arrangement of the vesicles in chains; (iii) iron uptake by the cell; (iv) iron transport into the magnetosome vesicle; and (v) controlled $\mathrm{Fe}_{3} \mathrm{O}_{4}$ (or $\left.\mathrm{Fe}_{3} \mathrm{~S}_{4}\right)$ biomineralization within the magnetosome vesicle [2]. However, the temporal order in which these steps occur was not known (e.g. whether the membrane vesicle forms before or after mineral nucleation).

\section{Roles of MamJ and MamK in magnetosome chain formation}

At the end of 2005, two studies by different groups of researchers reported on the roles of the proteins MamJ and MamK in magnetosome chain formation in 


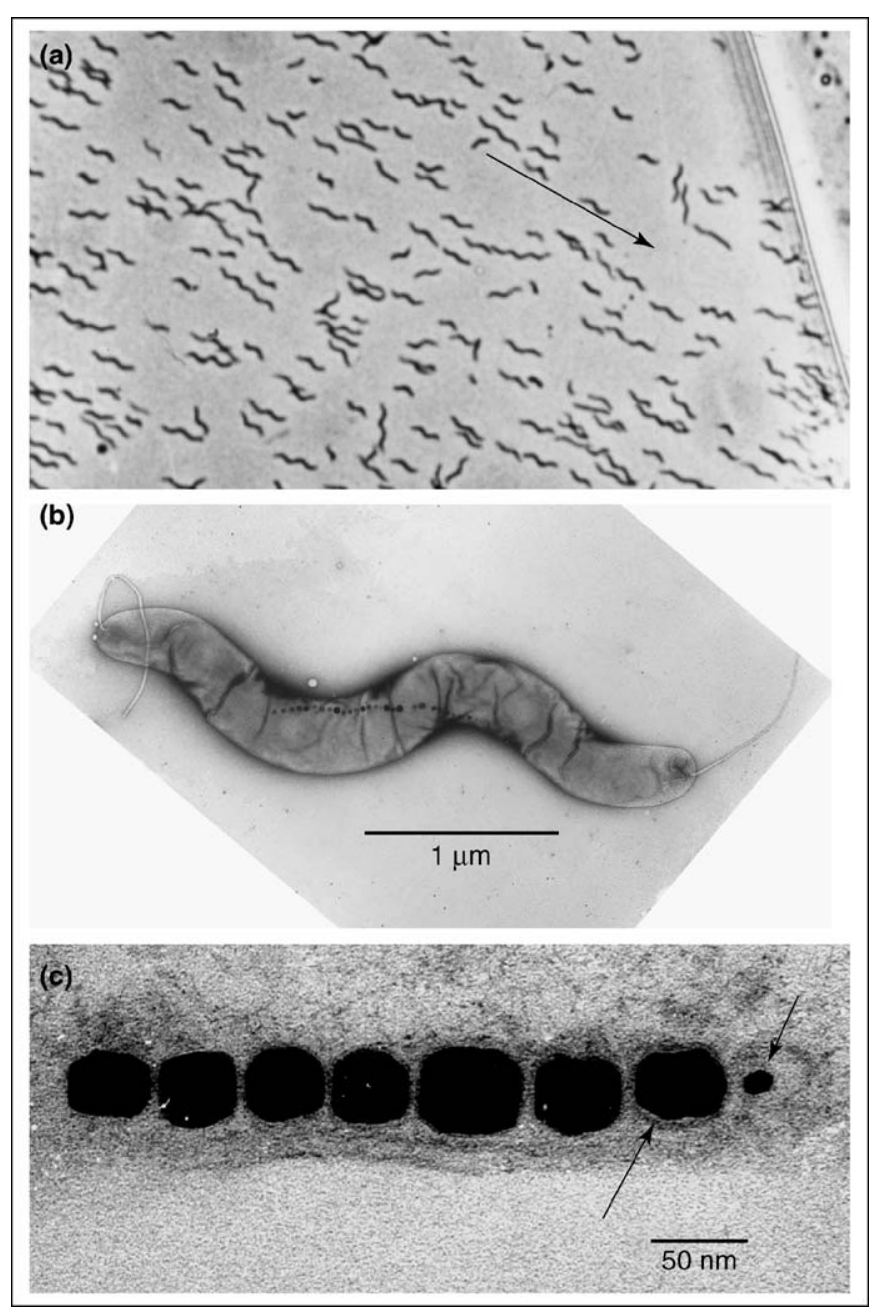

Figure 1. Magnetotaxis and magnetosomes. (a) Optical micrograph of Magnetospirillum magnetotacticum in a water drop aligned along the magnetic field (arrow). The bacteria are $\sim 3 \mu \mathrm{m}$ in length. (b) Transmission electron micrograph of a negatively-stained cell of $M$. magnetotacticum with a chain of magnetosomes and polar flagella. Part (b) reproduced, with permission, from Ref [16]. (C) (1989) Wiley-Liss. (c) Transmission electron micrograph of a thin section of a marine magnetotactic spirillum showing the magnetite crystals and magnetosome membrane (MM). The lower long arrow indicates a magnetosome in which the magnetite crystal is separated from the MM. The upper short arrow indicates a partially-filled MM vesicle. Part (c) reproduced, with permission, from Ref. [2]. (C) (2004) Nature Publishing Group.

Magnetospirillum species. Remarkably, the two groups converged on virtually identical approaches and methods.

Scheffel et al. [13] examined the role of the protein MamJ by generating a mamJ-deficient mutant, $\Delta$ mamJ, in $M$. gryphiswaldense. $\Delta$ mamJ produced magnetosomes that seemed to be identical to those in the wild type (WT) strain but they were organized as a cluster rather than as a chain. The authors genetically constructed an enhanced green fluorescent (EGFP) fusion protein with MamJ ( $m a m J-E G F P)$ to determine the intracellular location of MamJ. They found that MamJ was localized along a linear structure that extended from pole to pole in the cell, close to the cytoplasmic membrane in both WT and $\Delta$ mamJ cells. However, unlocalized MamJ was present in the cytoplasm of a non-magnetotactic deletion mutant that lacked all of the mam genes. This suggested that MamJ is not responsible for the linear structure alone but interacts with other mam gene products that make up the linear structure.
Electroncryotomography (ECT) of WT and $\Delta$ mamJ cells revealed a network of cytoskeletal filaments $\sim 3-4 \mathrm{~nm}$ thick. In WT cells grown under iron limitation, empty vesicles were associated with the filaments; however, in $\Delta$ mamJ cells, empty vesicles were dissociated from the filaments that seemed to be identical to those in WT cells. This convincingly shows that MamJ is involved in the connection of magnetosomes to the filaments and, therefore, in the construction of the magnetosome chain. Scheffel et al. [13] proposed a model for magnetosome chain assembly in which MamJ connects initially empty magnetosome vesicles to the cytoskeletal filaments. Magnetite crystal growth commences within the vesicles and the magnetosomes move towards the mid-cell where chains are formed. Scheffel et al. [13] also noted that mamJ is co-transcribed with mamK, and suggested that mamK encodes the cytoskeletal magnetosome filaments to which MamJ connects the magnetosome vesicles.

Serendipitously, Komeili et al. [14] reported their investigation of MamK almost simultaneously with that of Scheffel and co-workers. Komeili et al. [14] also used ECT to study magnetosome formation in M. magneticum strain AMB-1. They first convincingly showed that the magnetosome membrane vesicle originates as an invagination of the cytoplasmic membrane. In WT cells, magnetosomes are associated with networks of long filaments that run along the cytoplasmic membrane from the proximal to the distal pole, parallel to four or five magnetosomes along the chain. In addition to these long filament networks, a further seven filaments were found to flank the magnetosomes with no obvious spatial pattern. In nonmagnetic mutants that lack the $\operatorname{mam} A B$ gene cluster, no invaginations or filaments were found, which led to the hypothesis that MamK is responsible for the network of magnetosome filaments. To test this hypothesis, Komeili et al. [14] constructed an in-frame deletion of a mamK mutant. The mutant produced magnetosomes and responded to a magnetic field but did not have magnetosomes arranged in long, straight chains. Instead, the magnetosomes in $\Delta$ mamK were dispersed in the cell in small groups of two or three, separated by large gaps. Complementation of the $\Delta$ mamK mutant with mamK-GFP (MamK-green fluorescent protein fusion) resulted in restoration of the magnetosome chain and 6-nm magnetosome filaments that extended across the cell, following the cell curvature. Komeili et al. [14] concluded that MamK comprises the long magnetosome filaments that position magnetosome vesicles in chains.

\section{Concluding remarks and future perspectives}

The results reported in the two studies are complementary: both MamJ and MamK are responsible for magnetosome chain formation. Deletion of either mamJ or mamK leads to disruption of the chains but does not interrupt magnetosome formation. Both reports, together with previous work [15], show that magnetosome vesicles form first, followed by nucleation and growth of the magnetite crystals. However, there are differences, such as the organization of magnetosomes in $\Delta$ mamK compared to $\Delta$ mamJ mutants, and the fact that budding vesicles were only observed in $M$. magneticum and not in $M$. gryphiswaldense. What 
accounts for these differences? What initiates and mediates magnetosome vesicle formation? How do vesicles migrate and what causes them to accumulate at the mid-plane of the cell where they will be divided between daughter cells during cell division? What causes new magnetosomes to form at the ends of the inherited magnetosome chains? How are the magnetosomes oriented so that all the magnetosome crystals have a common crystallographic orientation? What are the functions of the products of other mam genes? These questions and many others must await future studies. Nevertheless, the results reported by Scheffel et al. [13] and Komeili et al. [14] converge in pointing out the path to understanding how magnetotactic bacteria construct their magnetic compass needles.

\section{Acknowledgements}

We thank D. Schüler for sharing unpublished results and discussions. D.A.B. is supported by NSF grant EAR-0311950.

\section{References}

1 Blakemore, R.P. (1982) Magnetotactic bacteria. Annu. Rev. Microbiol. $36,217-238$

2 Bazylinski, D.A. and Frankel, R.B. (2004) Magnetosome formation in prokaryotes. Nat. Rev. Microbiol. 2, 217-230

3 Frankel, R.B. et al. (1997) Magneto-aerotaxis in marine, coccoid bacteria. Biophys. J. 73, 994-1000

4 Bazylinski, D.A. et al. (1995) Controlled biomineralization of magnetite $\left(\mathrm{Fe}_{3} \mathrm{O}_{4}\right)$ and greigite $\left(\mathrm{Fe}_{3} \mathrm{~S}_{4}\right)$ in a magnetotactic bacterium. Appl. Environ. Microbiol. 61, 3232-3239

5 Schultheiss, D. and Schüler, D. (2003) Development of a genetic system for Magnetospirillum gryphiswaldense. Arch. Microbiology $179,89-94$
6 Matsunaga, T. et al. (1992) Gene transfer in magnetic bacteria: transposon mutagenesis and cloning of genomic DNA fragments required for magnetite synthesis. J. Bacteriol. 174, 2748-2753

7 Matsunaga, T. et al. (2005) Complete genome sequence of the facultative anaerobic magnetotactic bacterium Magnetospirillum $\mathrm{sp}$ strain AMB-1. DNA Res. 12, 157-166

8 Schübbe, S. et al. (2003) Characterization of a spontaneous nonmagnetic mutant of Magnetospirillum gryphiswaldense reveals a large deletion comprising a putative magnetosome island. J. Bacteriol. $185,5779-5790$

9 Ullrich, S. et al. (2005) A hypervariable 130-kilobase genomic region of Magnetospirillum gryphiswaldense comprises a magnetosome island which undergoes frequent rearrangements during stationary growth. J. Bacteriol. 187, 7176-7184

10 Grünberg, K. et al. (2001) A large gene cluster encoding several magnetosome proteins is conserved in different species of magnetotactic bacteria. Appl. Environ. Microbiol. 67, 4573-4582

11 Grünberg, K. et al. (2004) Biochemical and proteomic analysis of the magnetosome membrane in Magnetospirillum gryphiswaldense. Appl. Environ. Microbiol. 70, 1040-1050

12 Kürner, J. et al. (2005) Cryo-electron tomography reveals the cytoskeletal structure of Spiroplasma melliferum. Science 307, 436438

13 Scheffel, A. et al. (2005) An acidic protein aligns magnetosomes along a filamentous structure in magnetotactic bacteria. Nature 440, 110-114

14 Komeili, A. et al. (2005) Magnetosomes are cell membrane invaginations organized by the actin-like protein MamK. Science $311,242-245$

15 Komeili, A. et al. (2004) Magnetosome vesicles are present before magnetite formation, and MamA is required for their activation. Proc. Natl. Acad. Sci. U. S. A. 101, 3839-3844

16 Frankel, R.B. and Blakemore, R.P. (1989) Magnetite and magnetotaxis in bacteria. Bioelectromagnetics 10, 223-237 